\title{
AiMT
}

Advances in Military Technology

Vol. 13, No. 1 (2018), pp. 23-32

ISSN 1802-2308, eISSN 2533-4123

DOI 10.3849/aimt.01212

\section{Possibilities of Assessing Objectively a Flight Illusion Effect on a Pilot's Spatial Orientation}

\author{
J. Boril*, V. Smrz and R. Jalovecky \\ University of Defence in Brno, Czech Republic
}

\begin{abstract}
The manuscript was received on 1 September 2017 and was accepted after revision for publication on 22 March 2018.
\end{abstract}

\begin{abstract}
:
The pilot's spatial orientation, the same as the one of any other human, relies on a combination of stimuli coming from their audio-visual, vestibular and proprioceptive perception systems. However, these instinctive perceptual methods can easily fail while pilot is perceiving real changes of attitude, position or movement and they can also generate false illusions to each of the aforementioned parameters. This failure in a real flight is called "flight illusion" which can negatively affect the pilot's spatial orientation. The influence of a flight illusion on the pilot's spatial orientation can be assessed either subjectively or objectively. The aim of this paper is to demonstrate one of the possible objective assessments of the flight illusions influence on the pilot's spatial orientation using the flight data generated by an appropriate flight simulator which were analysed in MATLAB ${ }^{\circledR}$ software. The chosen method is based on a comparison of the digitalized standard instrument flight trajectories with the real flown trajectories in the time interval with potential flight illusion influence.
\end{abstract}

\section{Keywords:}

flight illusion, spatial orientation, flight data analysis, flight simulator, simulator-based training, flight safety

\section{Introduction}

Pilot, as any other human, when searching their position and moving in 3-D space, relies on a combination of stimuli coming from their audio-visual, vestibular and proprioceptive perception systems [1]. Nevertheless, most pilots while flying under good conditions with a clear visibility in daylight naturally tend to depend on visual stimuli (e.g. natural horizon) to maintain the needed spatial orientation. Conversely, at night or in clouds, when the natural horizon is not visible, human brain in its effort to main-

\footnotetext{
* Corresponding author: Department of Air Force and Aircraft Technology, Faculty of Military Technology, University of Defence in Brno, Kounicova 65, 66210 Brno, Czech Republic.

Phone: +420 9734439 49, e-mail: jan.boril@unob.cz
} 
tain the spatial and positional orientation inclines to the elementary vestibular and proprioceptive perceptions.

However, these instinctive perceptual methods can easily fail while perceiving real changes of attitude, position or movement and they can also generate false illusions to each of the aforementioned parameters. This failure in a real flight is called "flight illusion", the usual effect of which is a pilot's spatial disorientation [2].

By their potential, flight illusions are rated as one of gravest threats for flight safety [3]. As it results from analyses of available reports on air accidents being investigated in air forces of developed countries, a considerable number of those accidents were brought about by failure of the human factor, namely by failing to manage the effect of a flight illusion. The inability to respond to a flight illusion appropriately can lead to the pilot's spatial disorientation which may, under certain conditions, result in an air accident (usually with fatal consequences) [4].

The sources of flight illusions should be sought primarily in the physiology of human spatial orientation system that is not evolutionarily adapted for flying. All pilots, especially beginners, ought to be aware of this fact; therefore, the demonstration of its effects on the pilots' spatial orientation should be standardly included into the aero-medical training of military pilots $[5,6]$, as well as civilian ones, but no system solution to address this issue has been developed so far [7].

The possibility of demonstrating effects of flight illusions on military pilots' spatial orientation has been available in the Czech Air Force since 2004 when the Institute of Aviation Medicine in Prague put into operation GYRO IPT II, a new flight simulator and disorientation demonstrator. Based on this possibility and in compliance with the pertinent NATO standard, all pilots in the Czech Air Force are bound to pass through, once in 5 years, the lesson of demonstrating the effects of flight illusions as part of their aero-medical training [8]. New military pilots shall undergo this lesson prior to starting their practical flight training.

The demonstration is based on using of samples, predefined by the manufacturer, of flight illusions whose course is hard to change, whereas these demonstrations are not implemented into any complex flight missions. The opinion held by the authors is that a mere demonstration of a flight illusion does not use the full potential of the disorientation simulator.

A new approach developed by the authors is based on the flight illusion demonstration integrated into a more complex flight mission to enable the pilot to perceive an illusion as realistically as possible which mean unexpectedly with possibility to evaluate it objectively [9]. This part of methodology shall be an analysis of data obtained while executing the complex flight mission in order to assess objectively as much as possible the effects of a flight illusion on the pilot's spatial orientation.

\section{Types of Illusions Used in the Experiment}

People instinctively primarily rely on their visual/audible (sight $\&$ sound), vestibular (position and motion detection), and proprioceptor perceptions for their sense of direction and physical orientation cues. Quite naturally, during daytime flights, many aircraft pilots tend to rely heavily on visual cues from the horizon to maintain good spatial orientation. But at night or in a bad weather, the horizon is not visible and the human brain tends to fall back on the basic vestibular and proprioceptor senses in an attempt to maintain flight orientation and whole process of situational awareness $[10,11]$. 
However, these instinctive sensory methods can easily fail to detect real changes in altitude, attitude, or motion, and can even synthesize false cues for each. In addition, it has been proven that the semi-circular canals of the human ear are not very effective or reliable when serving as "change-in-velocity" sensors.

Surprisingly, the phenomenon known as "spatial disorientation in flight" can be induced by several simple physical factors. Many pilots may fail to pay enough attention or successfully recognize all the visual cues actually present. Some tend to rely too heavily on vestibular and proprioceptor perceptions in spite of having valid flight instrumentation readings available. Others are too slow, or fail to declare that a loss of one or more flight instruments, caused by an electrical or mechanical malfunction, has actually occurred. In this case is also important to take into account the aircraft like a whole system where dependability is crucial factor for pilot's spatial orientation [12].

\subsection{Coriolis Illusion}

Coriolis Effect is an illusion where the pilot experiences a tumbling sensation due to moving his or her head beyond the plane of rotation while the aircraft is performing a turn. An intense tumbling sensation is caused by the stimulation of the semi-circular canals in two or more axes due to multiple angular accelerations.

\subsection{Somatogyral Illusion}

Somatogyral is the false sensation of self-rotation caused by the inability of the semi-circular canals to accurately register sustained motion. Somatogyral illusion demonstrates how angular accelerations can lead to a misperception of aircraft rotation resulting in the aircraft entering a graveyard spin or spiral.

\section{GYRO IPT II Disorientation Simulator Description}

The GYRO IPT II simulator in Fig. 1 is an interactive, flexible and multi-functional training system designed for meeting all training requirements for all pilots, ranging from beginners to full professionals. It consists of a single-seat pilot cabin mounted on a sophisticated movable base. The cabin and software equipment is configured so as to be capable of demonstrating flight parameters and instrument boards of different types of airplanes (ranging from trainer planes, through helicopters to jet fighters). Included in the cabin is the pilot's seat with the harness arrangement, interactive feedback control system, instrument panel and realistic sound distribution.

The unique base featuring 6-level freedom of movement (4+2) allows demonstrating pitch, roll, yaw and elevation of the cabin, and also combinations elevation + pitch and elevation + roll. These combined movements are regulated by an advanced movement control system capable of generating instructions for precise multi-axial movements that creates the needed linear and angular accelerations. The accelerations can be generated both in the human detectable and undetectable (subliminal) zones for a virtual demonstration of the whole range of vestibular illusions in a high quality.

The complete simulator system is controlled from the instructor workplace which is located nearby and interconnected with the simulator by cables and signals. The instructor's workplace is equipped with all indispensable instruments such as computers, monitors, alternative control console, communication and recording equipment for controlling and monitoring all operational states and modes of the simulator. 


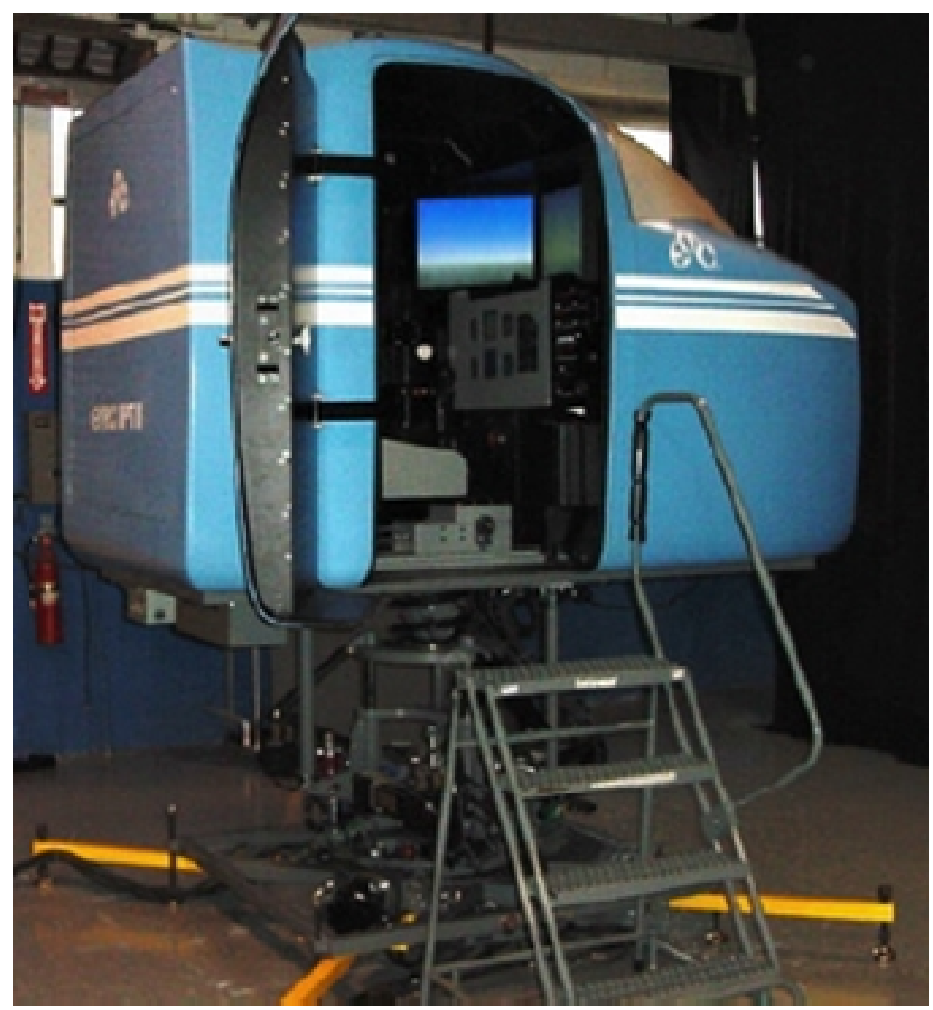

Fig. 1 GYRO IPT II cabin overview

\section{Data Analysis Methods and Techniques}

The possibilities of assessing the effects of a flight illusion on the pilot's spatial orientation depend primarily on the capabilities of the equipment performing the flight illusion demonstration and the instructor's level of competence and experience by operating of the device. The assessment method can be either subjective or objective, depending on the procedure to be used.

\subsection{Subjective Assessment Method}

This method depends first of all on the instructor's level of experience who, based on observing the pilot's behaviour (method of his / her piloting, analysis of the camera image, etc.) and monitoring data on the flight instruments, including the resulting trajectory, can subjectively define the effect of a flight illusion on the pilot's spatial disorientation, e.g. in the form of verbal debriefing. However, this method of assessment strongly depends on the instructor's personality and most likely the conclusions would be different, if done by another instructor. In such a case the possibility of conducting a mutual comparison of results produced by individual pilots becomes very complicated. 


\subsection{Objective Assessment Method}

In contrast to the subjective assessment, this method depends principally on the technical parameters of the device (flight simulator) and quality of the applied method of assessment. First of all, the prime condition rests in the availability of data files that contain the record of defined flight parameters. Included among the fundamental flight parameters, as needed for assessing the effect of an illusion, are:

- the record of flight trajectory in horizontal plane (in real geographic coordinates),

- the time flow depiction of recorded changes of flight altitude,

- the time flow depiction of recorded changes of magnetic heading.

The presented data shall be kept available in a digital form, ideally of at least $1 \mathrm{~Hz}$ record frequency so as to allow being processed by means of software instruments, see Chapter V. The flight parameters obtained objectively can be compared to a defined standard or to each other. In order to assess the effects of a flight illusion on the pilot's spatial disorientation objectively, the authors have developed a procedure as shown in the following paragraphs.

The authors created a complex flight mission as described in Chapter III which uses standard instrument flight procedures (i.e. standard instrument departures and approaches) which the pilot can do without the instructor's instruction while undertaking it. To minimize the instructor's influence on performing the flight mission, all information shall be obtained by the mission briefing and from the instrument chart placed inside the cockpit.

This flight mission was flown on the flight simulator several times as precisely as possible to digitally record the ideal flight trajectories and changes in defined parameters (altitude and magnetic heading). These records were averaged and adapted by software to eliminate the influence of inaccuracies in piloting and to allow for creating an ideal trajectory standard and changes of defined parameters in a digital form so as to be used for the comparison with real trajectories and flight parameters by the missions under the effect of an illusion.

The records of digital data obtained from real flights were compared to the above-mentioned ideal trajectory standard and changes, so as to achieve the result in the form of values showing deviations from the ideal trajectory caused under the influence of an illusion that should characterize objectively the effect of the applied illusion on the pilot's spatial orientation and his capability to keep it under control.

As described in Chapter III, GYRO IPT II disorientation simulator is able to create flight illusion in specific conditions and specific time. The authors have set up the simulator to create flight illusion after final turn to the ILS (Instrument Landing System) in flight time 540 seconds. From this point, authors start to collect the data for the future evaluation. The data are stored for 3 minutes when the pilot according to previous study should be influenced by the illusion. Only those data are taken into consideration for processing and evaluating in MATLAB ${ }^{\circledR}$. The whole flight task from the take-off until landing is influenced by simple pilot-tracking error between ideal flight trajectory and real flight - pilot's ability to keep required track, however, this pilot-tracking error is not a subject matter of this study. The deviation in the area of interest is a sum of pilot-tracking error and flight illusion influence which is much bigger (calculated deviation values) than pilot-tracking error that means determinative for processing and evaluation. 


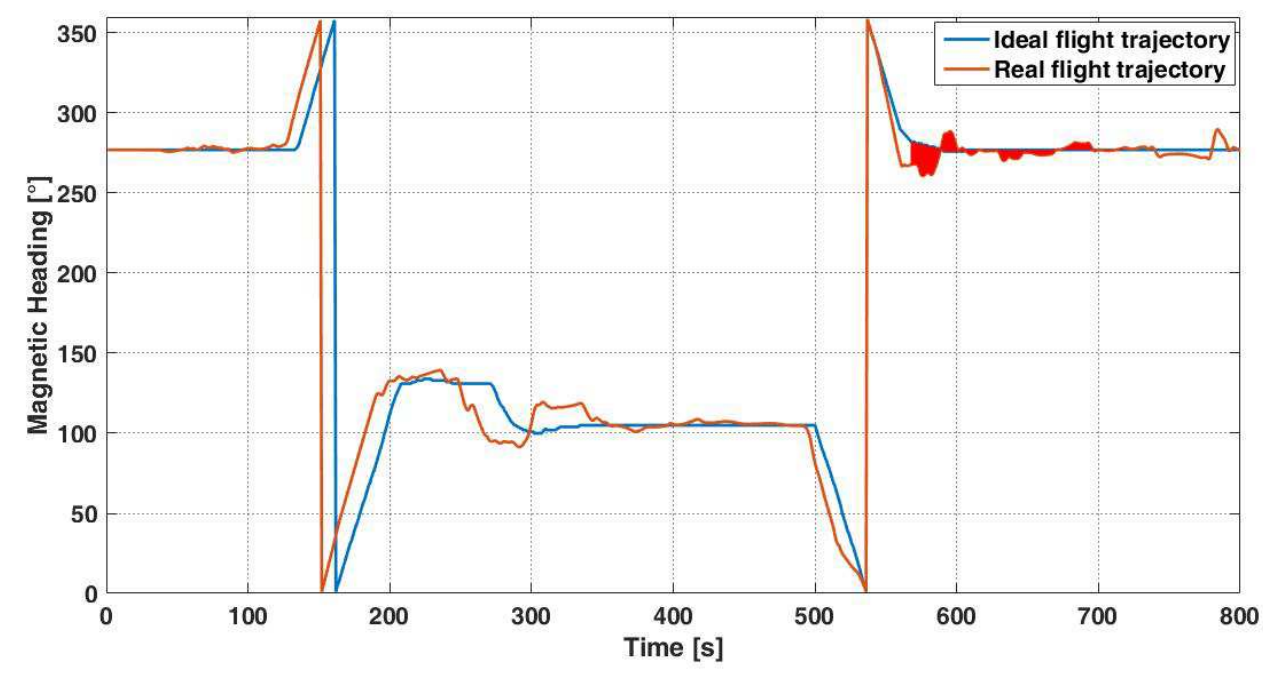

Fig. 2 Deviation in horizontal plane

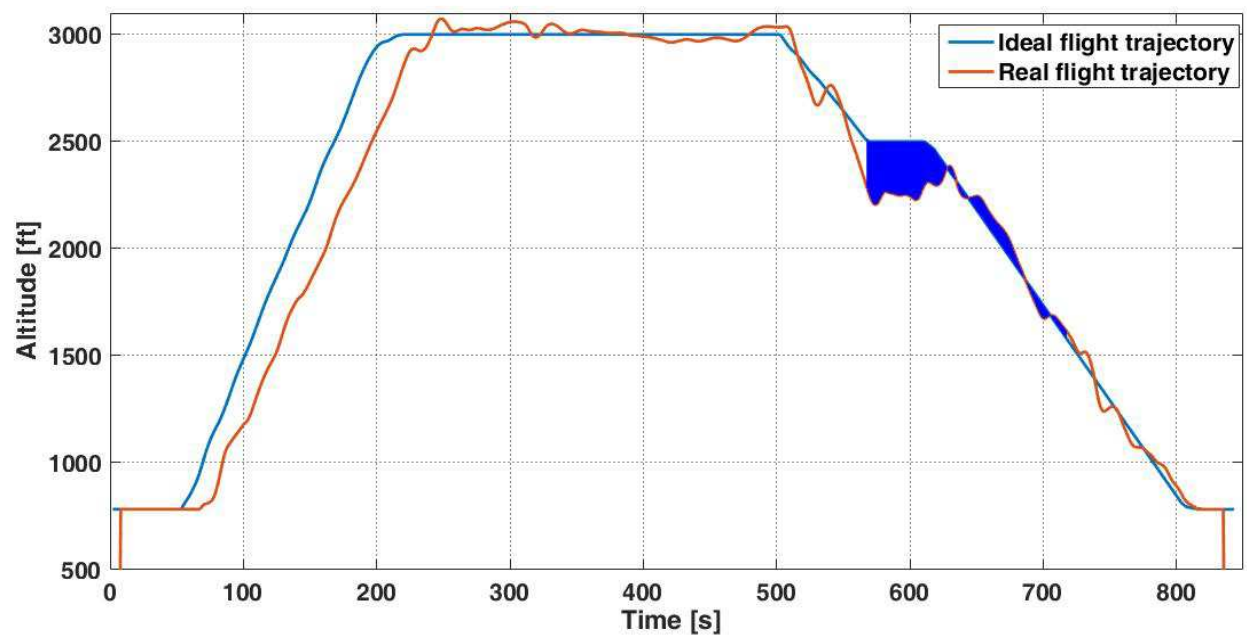

Fig. 3 Deviation in vertical plane

The authors analysed two types of deviations, i.e. in horizontal plane (Fig. 2) based on the comparison of the time flow depiction of recorded changes of magnetic heading (identical with ILS LLZ course, as no cross wind was simulated), and in vertical plane (Fig. 3) based on the comparison of the time flow depiction of recorded changes of flight altitude by the real (influenced by illusion) and ideal flight mission.

Bearing in mind the nature and differences of individual flights, the comparison of digital records of real and ideal flight trajectory in geographic coordinates in Fig. 4 is not expressed in numerical values. Nevertheless, the visualized record of a real trajectory was used for a subjective assessment of the effect of a flight illusion on the flight trajectory that was in some cases used as a complement to assessing objectively the influence of a flight illusion on the pilot's spatial orientation. 


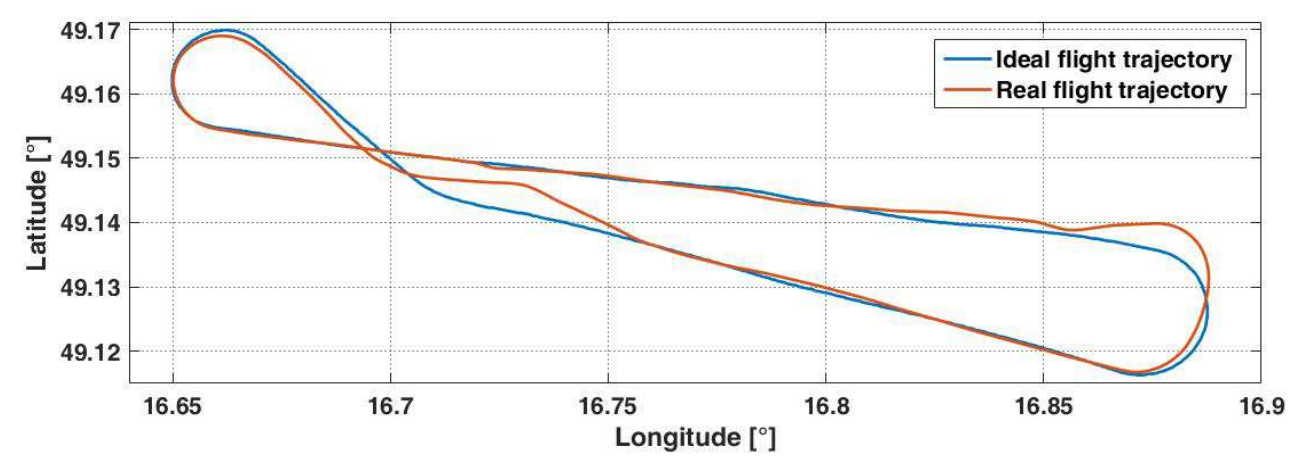

Fig. 4 Trajectory in geographic coordinates

The comparison of digital records has resulted in specific deviation values (Area values in Fig. 5) in horizontal directions - expressed in angular degrees and vertical directions - expressed in feet obtained by adding up the deviations perpendicular to the ideal trajectory in $1 \mathrm{~s}$ time step throughout the entire period of the monitored interval $(180 \mathrm{~s})$ that was set upon previous test flights done prior to conducting the experiments. This is an interval ranging from the beginning of the moment when the illusion starts to take effect (in direct relation to the principle of disorientation simulator operation) to the moment when the illusion effect is considered worn off at $95 \%$ of pilots participating in the experiment (Fig. 5).
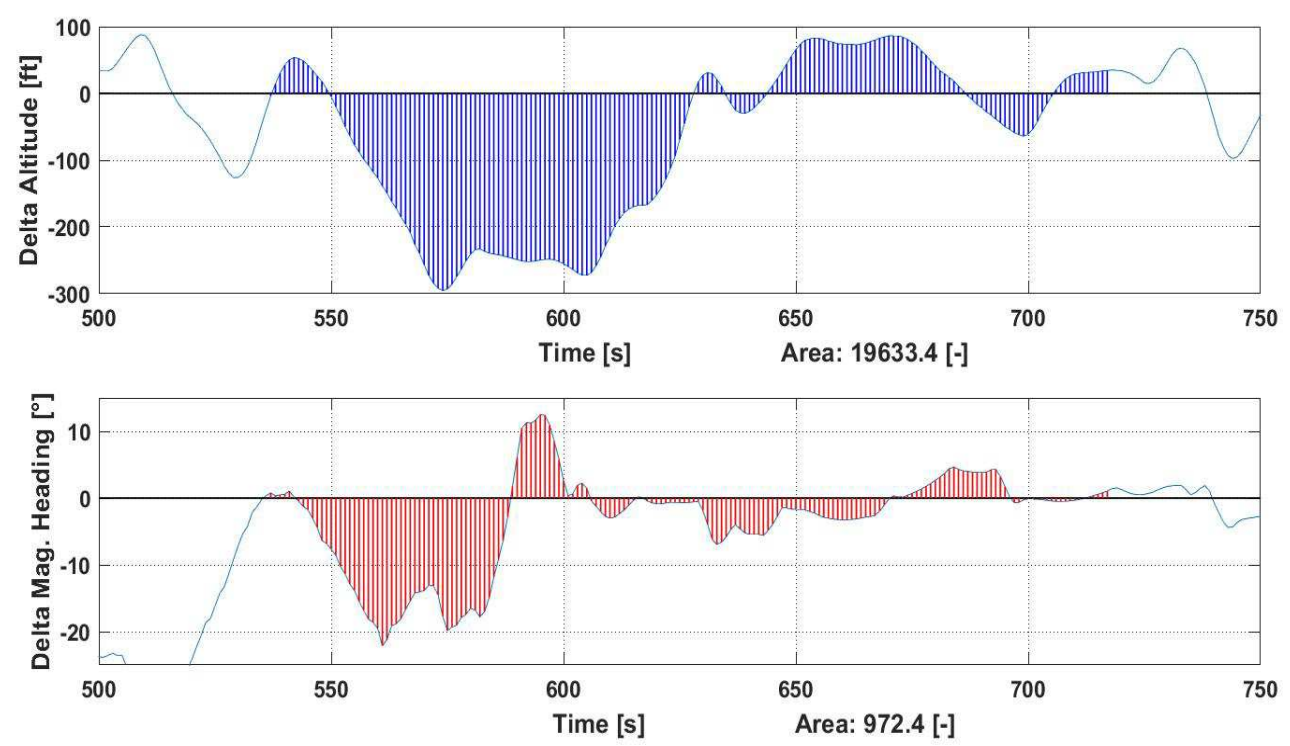

Fig. 5 Deviations in horizontal and vertical directions (numerical values - Area)

The resulting two types of deviations can be compared either separately; in order to be able to compare one value, or the pertinent horizontal and vertical deviations can be multiplied with each other, the result of which will be one value objectively characterizing the effect of a flight illusion on the pilot's spatial orientation in 3D within a defined time interval (with an unreal quantity of angular degree $x$ feet). 
All the above-stated values can be mutually compared both to each other and to values averaged for setting the sought dependencies or correlations with eventual measurements of the selected pilot's biometric parameters [13, 14] (heart rate, stress indicators, stability of the vestibular system, etc.).

\section{Program Description for the Objective Assessment Method}

Processing data files obtained from individual flights that also contain the flight section with an evoked illusion is performed in the MATLAB ${ }^{\circledR}$ development environment. The entire course of data processing is depicted in the form of a flowchart, see Fig. 6. To be able to compare the ideal flight trajectory with the illusion which influenced flight trajectories, the authors had to create a "referential" flight file to which individual flights would be subsequently compared.

As soon as it is read and depicted in a graphic form, the operators may choose the desired tested flight from a menu. The following step rests in "synchronizing" data files by the beginning of the potential moment when the illusion was evoked. Thanks to the position of the selected airport, it takes place just when the aircraft heading is changed from $0^{\circ}$ to $360^{\circ}$ (see Fig. 2). In terms of programming, this solution, i.e. heading commutation, is of course the easiest option. At the moment when data are synchronized, the selected flight can be depicted in the referential data (see Figs. 2-4). The next step lies in calculating the area of deviation from heading and flight altitude, see the hatched section in Figs. 2 and 3. The graph, as generated by the pattern of Fig. 5, gives a better overview of the deviations where the deviation sections are magnified and the value of calculated area is shown.
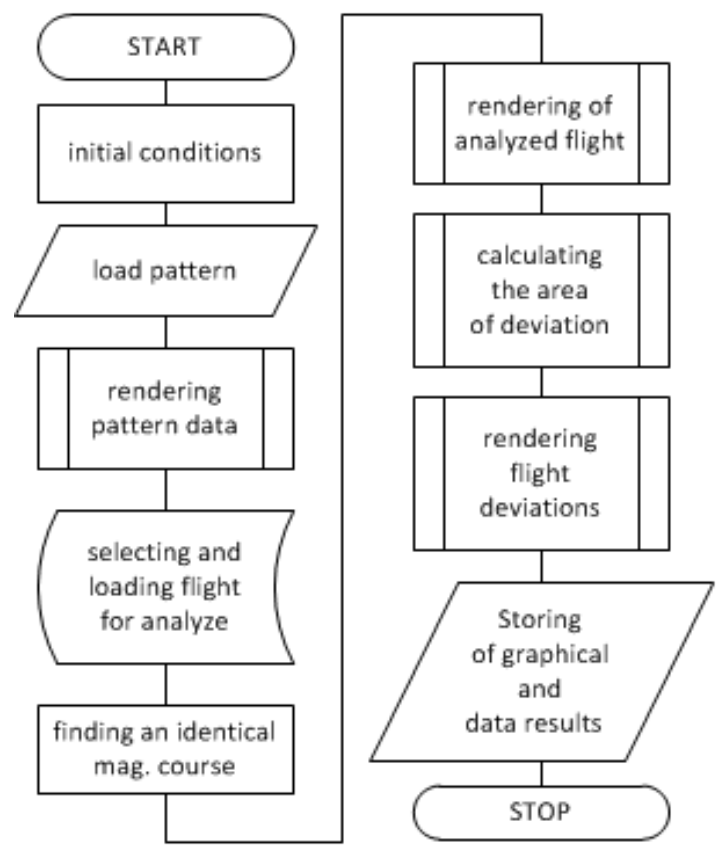

Fig. 6 Flowchart for processing data obtained from in-flight measurements of flight illusions 
At the end of the analysis, all graphic outputs are saved in .png or .fig file formats. The values of the calculated area are entered into the text file by adding the lines of calculated data with the identification of the analysed flight. This file is also used for further research based on comparing individual flights.

\section{Conclusion}

As mentioned in the introduction, the influence of some flight illusions may very seriously affect the flight safety. Because of this knowledge, the pilots in many countries absolve the flight illusion demonstrations by using sophisticated means, like disorientation simulators to be aware of its influence. The authors of this paper are confident that these tools are suitable not only to demonstrate the flight illusion influence, but that they can be successfully used for complete flight missions with illusion incorporated into them to demonstrate the flight illusion influence as close to reality as possible (i.e. unexpectedly) to train the pilot's situational awareness.

As an added value, such missions can be used for an objective assessment of the flight illusion influence on the pilot's spatial orientation (which belongs to the situational awareness) by means of the assessment of the flight trajectory deviation as described in the paper. The mission results (calculated deviation values) from certain sample of pilots can be used for statistical assessment of the flight illusion influence between the pilots in one sample (i.e. the same flight experiences), between different pilots' samples (i.e. different flight experiences) or the influence of different type of illusions (i.e. between Somatogyral or Coriolis illusion).

According to the obtained data it seems that the described experiments can lead to more effective utilization of the disorientation simulator with the goal to increase flight safety and to encourage research activities connected with this goal. The objective assessment method will be beneficial for the future when the authors intent to measure more pilots with different skills and experience with flight illusions. Down to this processing and evaluation methods, the authors assume they will have a list of pilots for whom the focused spatial orientation training will be possible directly, tailor-made to individual persons whose inner ear sensitivity varies.

\section{Acknowledgement}

The work presented in this paper was also supported by the Czech Republic Ministry of Defence - University of Defence Development Program "Support for operations of the Czech Air Force in local conflicts" and "Research of sensor and control systems to achieve battlefield information superiority".

\section{References}

[1] CHEUNG, B. Spatial Disorientation: More than just Illusion. Aviation Space and Environmental Medicine, 2013, vol. 84, no. 11, p. 1211-1214. DOI 10.3357/ ASEM.3657.2013.

[2] BENSON, A.J. Spatial Disorientation - Spatial Aspects, Common Illusion and Motion Sickness. In ERNSTING, J., NICHOLSON, A.N. and RAINFORD, D.J. (eds.) Aviation Medicine. Oxford: Butterworth Heinemann, 1999, p. 419-471. 
[3] HOLMES, S.R. et. al. Survey of Spatial Disorientation in Military Pilots and Navigators. Aviation Space and Environmental Medicine, vol. 74, no. 9, 2003, p. 957-965. ISSN 0095-6562.

[4] GIBB, R., ERCOLINE, B. and SCHARFF, L. Spatial Disorientation: Decades of Pilot Fatalities. Aviation Space and Environmental Medicine, 2011, vol. 82, no. 7, p. 717-724. DOI 10.3357/ASEM.3048.2011.

[5] PETRU, A. and FRANTIS, P. Concept of Improving Pilot's Sensory Illusion Resistance. In International Conference on Military Technologies 2015, Brno: University of Defence, 2015. DOI 10.1109/MILTECHS.2015.7153654.

[6] KALLUS, K.W. and TROPPER, K. Evaluation of a Spatial Disorientation Simulator Training for Jet Pilots. International Journal of Applied Aviation Studies, 2004, vol. 4, no. 1, p. 45-56. ISSN 1939-0300.

[7] KLYDE, D.H., LAMPTON A.K. and SCHULZE, P.C. Development of Spatial Disorientation Demonstration Scenarios for Commercial Pilot Training. In AIAA Modeling and Simulation Technologies Conference, 2016, San Diego: AIAA. ISBN 978-1-5108-2734-9.

[8] SAZEL, M., PAVLIK, J., PETRICEK J. and SEDLATY, Z. Spatial Disorientation Training. In 53 ${ }^{\text {rd }}$ International Congress of Aviation and Space Medicine, Warsaw, 2005.

[9] BORIL, J. SMRZ, V. LEUCHTER, J. and BLASCH, E. Increasing Flight Safety Using Flight Sensory Illusions on a Spatial Disorientation Simulator. In $35^{\text {th }}$ IEEE/AIAA Digital Avionics Systems Conference (DASC), 2016, Sacramento: IEEE, p. 1-5. DOI 10.1109/DASC.2016.7778095.

[10] BLASCH, E. PACES, P. and LEUCHTER, J. Pilot Timeliness of Safety Decisions Using Information Situation Awareness. In $33^{\text {rd }}$ IEEE/AIAA Digital Avionics Systems Conference (DASC), 2014, Colorado Springs: IEEE, p. 7D6-17D6-9. DOI 10.1109/DASC.2014.6979525.

[11] KOZUBA J. and PILA, J. Selected Elements Influencing Pilot Situational Awareness. Advances in Military Technology, 2015, vol. 10, no. 2, p. 45-55. ISSN 1802-2308.

[12] WOCH, M., ZIEJA, M. and TOMASZEWSKA, J. Analysis of the Time between Failures of Aircrafts. In $2^{\text {nd }}$ International Conference on System Reliability and Safety (ICSRS), 2017, Milan: IEEE, p. 112-118. DOI: 10.1109/ICSRS.2017. 8272805.

[13] REGULA, M. et. al. Study of Heart Rate as the Main Stress Indicator in Aircraft Pilots. In Proceedings of the $16^{\text {th }}$ International Conference on Mechatronics, Mechatronika, 2014, Brno: IEEE, p. 639-643. DOI 10.1109/MECHATRONIKA. 2014.7018334.

[14] SOCHA, V. et. al. Basic Piloting Technique Error Rate as an Indicator of Flight Simulators Usability for Pilot Training. International Review of Aerospace Engineering, 2016, vol. 9, no. 5, p. 162-172. DOI 10.15866/irease.v9i5.10749. 